\title{
CARIFTAA CSME: ¿DE ZONA DE LIBRE COMERCIO A LA UNIÓN ECONÓMICA Y POLIITICA?
}

\author{
CARIFTA TO CSME: FROM FREE ZONE \\ TO ECONOMIC AND POLITICAL UNION?
}

\author{
Elizabeth Abigail Sampson ${ }^{1}$
}

\section{RESUMEN}

Los esquemas de integración regional fortalecen vínculos entre los países miembros (regionalismo cerrado). Muchas veces, los países miembros son países vecinos. A la vez, la colaboración con otros esquemas promueve la solidaridad. No obstante, los esquemas de integración están aquejados por las asimetrías. La Comunidad Caribeña (CARICOM) es un esquema de integración regional que también enfrenta muchos retos; pero hay potencial para llegar al mismo nivel de integración como la Unión Europea. Este artículo examina la evolución, los retos, y las oportunidades de la CARICOM y ofrece varias recomendaciones.

Palabras claves: Integración regional, solidaridad, asimetrías, CARICOM, CSME, Caribe.

\begin{abstract}
Regional integration schemes strengthen links between member countries (closed regionalism) which are often neighbouring countries. At the same time, collaboration with other schemes can promote solidarity. Nevertheless, integration schemes are affected by asymmetries. The Caribbean Community (CARICOM) is a regional integration scheme which faces many challenges but has the potential to reach the same level of regional integration as the European Union. This article examines the evolution, challenges, asymmetries and opportunities of CARICOM and offers recommendations.
\end{abstract}

Key words: regional integration, solidarity, asymmetries, CARICOM, CSME, Caribbean.

1 Doctoranda en Doctorado de Estudios Latinoamericanos con énfasis en pensamiento, Universidad Nacional de Costa Rica, Costa Rica, 2013-2015. Maestría en Español, The University of the West Indies, St. Augustine, Trinidad and Tobago, West Indies, 2009-2012. Licenciatura en Administración Pública Nueva (BSc. Public Sector Management (minor in Spanish), 2004-2007, The University of the West Indies, St. Augustine, St. Augustine, Trinidad and Tobago, West Indies. elizsamp@yahoo. com elizsamp@gmail.com 


\section{Introducción}

Actualmente, muchas naciones intercambian sus bienes y servicios. Ninguna tiene todos los recursos naturales y humanos. Con la llegada de la globalización, existe la necesidad de intercambiar y establecer mercados y socios. Ningún ser humano es una isla y puede existir sin la interdependencia. La integración regional es un gran beneficio de cualquiera región en el ámbito cultural, económico y político. Dunnia Marín C. (2013), en su libro El abordaje de las asimetrías estructurales en los esquemas de integración regional, destaca la definición de la integración regional por el autor Tamames: “...es un proceso mediante el cual dos o más mercados se unen para formar uno sólo de una dimensión más idónea, por lo que se procura acoplar estructuras.” (Marín, 2013, 26) Los Estados miembros de CARICOM tienen retos y oportunidades en el mundo globalizado. Este artículo va a tratar de definir el Caribe, destacar los beneficios, los niveles, los retos, las oportunidades de integración regional y dar recomendaciones al esquema de integración regional, CARICOM, para llegar al nivel de la Unión Europea (UE) y la Organización de Estados del Caribe Oriental (OECO).

\section{La experiencia del Caribe en la integración}

Norman Girvan (2001), en su artículo "Reinterpreting the Caribbean", ofrece varias interpretaciones del Caribe. En la bibliografía hispana, se entiende como solo las islas hispanohablantes y las Antillas, sino el archipiélago entero. Por otro lado, el Caribe está interpretado, según una categoría sociohistórica, por los pensadores caribeños. Es una zona cultural con un legado de esclavitud y sistema de plantación (Girvan, 2001). El Caribe consiste en todas las islas, partes del continente y las comunidades diásporas (Girvan, 2001) De hecho, Girvan (2001) entiende los problemas o los retos que muchos individuos enfrentan y utiliza una tabla que esclarece las dudas. 


\section{Tabla 1}

Varias interpretaciones del Caribe

\begin{tabular}{|c|c|c|c|}
\hline Nombre & Ámbito & Caracterización & Instituciones \\
\hline Cuenca del Caribe & Continente e islas & $\begin{array}{l}\text { Geopolítica/hegemó- } \\
\text { nica }\end{array}$ & $\mathrm{CBI}$ \\
\hline El Gran Caribe 1 & Continente e islas & $\begin{array}{l}\text { Geoeconómica/coo- } \\
\text { perativa }\end{array}$ & ACS \\
\hline El Gran Caribe 2 & Continente e islas & $\begin{array}{l}\text { Geosocial y antihege- } \\
\text { mónica }\end{array}$ & CRIES, Foro civil \\
\hline Caribe plantación & $\begin{array}{l}\text { Islas, tres Guayanas y } \\
\text { comunidades negras } \\
\text { en el continente }\end{array}$ & $\begin{array}{l}\text { Etnohistórica/antihe- } \\
\text { gemónica }\end{array}$ & CSA \\
\hline Caribe insular & $\begin{array}{l}\text { Islas, tres Guayanas y } \\
\text { Belice }\end{array}$ & Etnohistórica & CDCC,ACE, CPDC \\
\hline $\begin{array}{l}\text { Caribe del } \\
\text { CARICOM }\end{array}$ & $\begin{array}{l}\text { Estados anglófonos, } \\
\text { Surinam, Montserrat }\end{array}$ & $\begin{array}{l}\text { Cooperativa econó- } \\
\text { mica, vínculos fuer- } \\
\text { tes culturales y lin- } \\
\text { güísticos }\end{array}$ & CARICOM \\
\hline Caribe del ACP & $\begin{array}{l}\text { CARICOM, Repúbli- } \\
\text { ca Dominicana, Haití }\end{array}$ & $\begin{array}{l}\text { Negociación/ neoco- } \\
\text { lonial en transición }\end{array}$ & CARIFORUM \\
\hline
\end{tabular}

Nota: Norman Girvan (2001, p. 2).

Para este artículo, se utilizará la definición de Girvan: los países caribeños son los quince miembros de la CARICOM que son los Estados anglófonos (Antigua y Barbuda, Bahamas, Barbados, Belice, Dominica, Granada, Guyana, Jamaica, Montserrat, Saint Kitts y Nevis, Santa Lucía, San Vicente y Granadinas y Trinidad y Tobago), Haití y Surinam.

No hay duda de que con la unidad, hay fortaleza y la integración regional es un mecanismo para fortalecer el comercio en el mundo. Usualmente, la integración regional ocurre entre los países vecinos. En este caso, los que comparten el mismo mar: el mar Caribe. Marín (2013) destaca el propósito de la integración regional propuesto por el autor Bela Balassa. "...considerada como un proceso, implica medidas orientadas a abolir la discriminación entre unidades económicas pertenecientes a diferentes Estados nacionales; ... la integración viene a caracterizarse por la ausencia de varias formas de discriminación entre economías nacionales" (p. 1). En CARICOM existe la no discriminación: el libre movimiento de bienes, servicios y personas. De hecho, el Artículo 7 del Tratado 
Revisado de Chaguaramas establece este derecho para lograr su último objetivo que es la unión económica y monetaria.

Existen diferentes niveles de integración regional. Max Fontes (2000), en su artículo "Mercosul and Supranationality: How to Overcome Brazilian Constitutional Obstacles", menciona los niveles de integración según la teoría de Balassa. El primer nivel es la zona de libre comercio, en la que hay la eliminación de los impuestos de importación y las restricciones cuantitativas entre socios (Fontes, 2000). Los bienes necesitan tener un certificado de origen que indique el país de origen para prevenir la desviación en el comercio (Fontes, 2000). Por ejemplo, los bienes entran con el arancel externo más bajo (Fontes, 2000). Girvan (2009) destaca la evolución de CARICOM. El primer nombre era CARIFTA (Asociación de Libre Comercio del Caribe) y se lanzó en 1965-1967 (Girvan, 2009).

La segunda etapa de integración regional es la unión aduanera (Fontes, 2000). En esta, como en la zona de libre comercio, hay la eliminación de obstáculos al libre comercio. Los países miembros en este nivel de integración regional están de acuerdo con el mismo arancel externo. Elimina la necesidad de los certificados de origen en las fronteras internas (Fontes, 2000). Es decir, hay el libre movimiento de bienes. El segundo nivel de integración regional en la CARICOM era la Comunidad Caribeña y Mercado Común, de hecho, dentro de este nivel de integración, en 1973, los grupos líderes de la CARICOM decidieron fusionar el segundo nivel con el tercero, el mercado común, que una unión aduanera en la que hay los factores de producción de capital y recursos (Fontes, 2000). Pueden existir diferentes reglamentos nacionales (como en la zona de libre comercio) o un reglamento común (como en la unión aduanera) (Fontes, 2000).

La última etapa de integración regional es considerada muchas veces como la unión económica (Fontes, 2000). Esta no es solo un mercado común, sino que también tiene un alto grado de coordinación o aun la unificación de las más importantes áreas de política económica, la regulación del mercado, políticas macroeconómicas, monetarias y de redistribución de ingresos (Fontes, 2000). En esta etapa, hay una política comercial común para los terceros países y políticas externas en los factores de producción. Este es el nivel de integración regional que la CARICOM deseó lograr y, en el cambio de nombre, se señaló su intento. Girvan (2009) menciona que desde 1989 los países miembros de la CARICOM quisieron lograr este objetivo. Paraello, el Mercado Común y Economía Únicos (CSME) necesita examinar los desafíos y las asimetrías que la CARICOM enfrenta. 
Aunque Max Fontes (2000) y Dunnia Marín (2013) mencionan que la última etapa de integración regional es la unión económica, esta última investigadora opina que la etapa final es la unión política y económica, es decir, que los países miembros en este nivel tienen instituciones supranacionales y comunes como un Parlamento, una Corte de Justicia, un Banco Central y un sistema de seguridad, transporte aéreo y marítimo. Sin embargo, hay una implicación seria aquí: los Estados miembros necesitan intercambiar su soberanía para la supranacionalidad y este es el gran reto de muchos esquemas de integración regional en el mundo. Muchas veces los grupos líderes de los países miembros no ven los beneficios de esta etapa de integración y la ciudadanía reclama que no hay intercambio. De hecho, este asunto puede provocar malas relaciones de poder entre los Estados miembros, como las que se suscitaron entre los primeros ministros de Jamaica y Trinidad y Tobago, que provocaron el fin de la Federación de las Antillas en 1962.

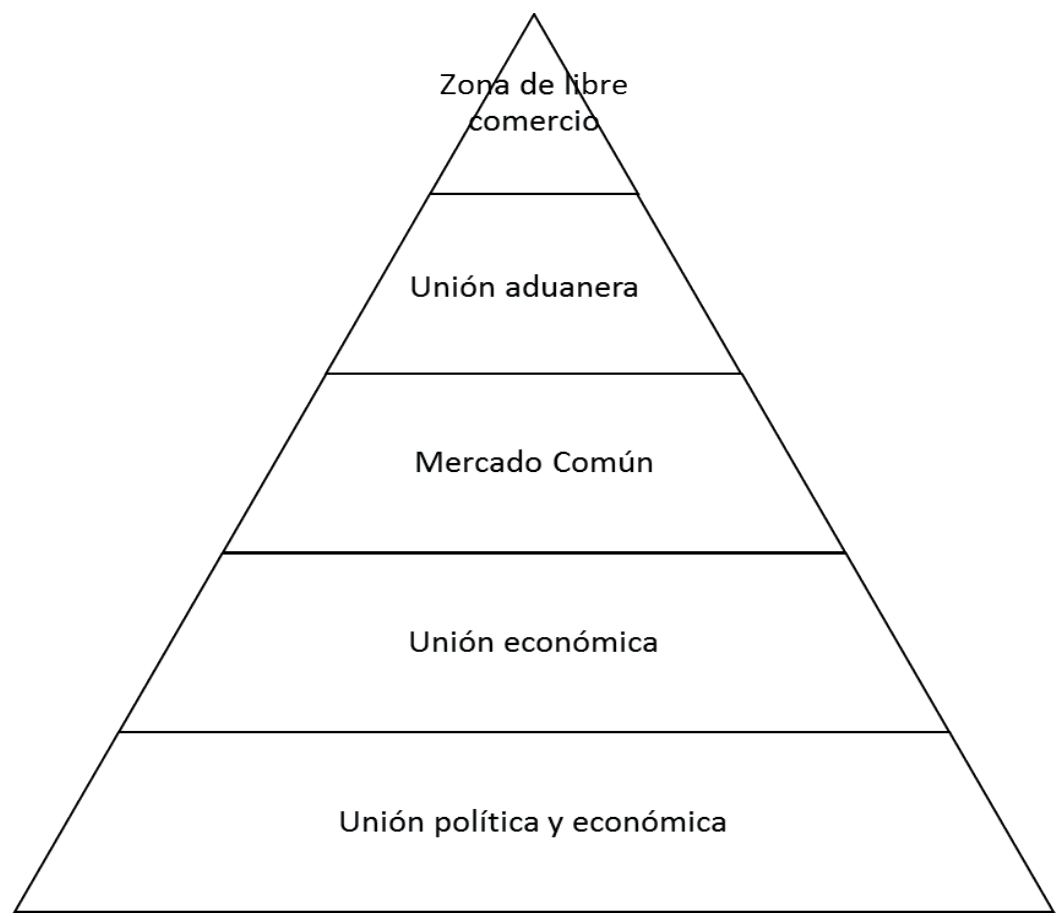

Figura 1. Niveles de integración regional. Elaboración propia. 
Tabla 2

Etapas hacia la unión monetaria

\begin{tabular}{|l|l|l|l|l|}
\hline $\begin{array}{c}\text { Zona de libre } \\
\text { comercio }\end{array}$ & \multicolumn{1}{|c|}{$\begin{array}{c}\text { Unión } \\
\text { aduanera }\end{array}$} & \multicolumn{1}{c|}{$\begin{array}{c}\text { Mercado } \\
\text { Común }\end{array}$} & \multicolumn{1}{c|}{$\begin{array}{c}\text { Unión } \\
\text { económica }\end{array}$} & \multicolumn{1}{c|}{$\begin{array}{c}\text { Unión } \\
\text { monetaria }\end{array}$} \\
\hline $\begin{array}{l}\text { Libre comer- } \\
\text { cio de bienes } \\
\text { y servicios }\end{array}$ & $\begin{array}{l}\text { Libre comer- } \\
\text { cio de bienes } \\
\text { y servicios }\end{array}$ & $\begin{array}{l}\text { Libre comercio } \\
\text { de bienes y } \\
\text { servicios }\end{array}$ & $\begin{array}{l}\text { Libre comercio de } \\
\text { bienes y servicios }\end{array}$ & $\begin{array}{l}\text { Libre comercio de } \\
\text { bienes y servicios }\end{array}$ \\
\hline & $\begin{array}{l}\text { Arancel ex- } \\
\text { terno común }\end{array}$ & $\begin{array}{l}\text { Arancel externo } \\
\text { común }\end{array}$ & $\begin{array}{l}\text { Arancel externo } \\
\text { común }\end{array}$ & $\begin{array}{l}\text { Arancel externo } \\
\text { común }\end{array}$ \\
\hline & & $\begin{array}{l}\text { Libre movilidad } \\
\text { de personas y } \\
\text { capitales }\end{array}$ & $\begin{array}{l}\text { Libre movilidad de } \\
\text { personas y capitales }\end{array}$ & $\begin{array}{l}\text { Libre movilidad de } \\
\text { personas y capitales }\end{array}$ \\
\hline & & $\begin{array}{l}\text { Coordinación de las } \\
\text { políticas macroeco- } \\
\text { nómicas con conver- } \\
\text { gencia macroeconó- } \\
\text { mica vinculante. }\end{array}$ & $\begin{array}{l}\text { Coordinación de las } \\
\text { políticas macroeco- } \\
\text { nómicas con conver- } \\
\text { gencia macroeconó- } \\
\text { mica vinculante. }\end{array}$ \\
\hline & & & $\begin{array}{l}\text { Fijación de los tipos } \\
\text { de cambio y sustitu- } \\
\text { ción de monedas na- } \\
\text { cionales por moneda } \\
\text { común. }\end{array}$ \\
\hline
\end{tabular}

Nota: Barboza, Jorge (2010).

\section{Perspectivas de integración regional}

Existen diferentes perspectivas de integración regional. A través de una sola teoría no es posible explicar los esquemas de integración regional. Las teorías/ perspectivas de integración regional son: neofuncionalista, intergubernamentalista, realista y liberal. Thanavat Pimoljinda (2010), en su artículo "Theoretical Discussion on Regional Integration: EU-ASEAN perspective", hace un buen trabajo al destacar varias opiniones teóricas de integración regional.

En primer lugar, los grupos neofuncionalistas creen que la cooperación económica puede diseminar a otras áreas y provocar más integración. (Pimoljinda, 2010). Es decir, si cada nivel de integración regional es exitoso, sirve como forma de motivación para continuar la integración y elevarla a nivel regional. Esta es la razón por la que los miembros de la CARICOM decidieron transformarse de zona de libre comercio en los años tempranos y quieren transformarla en una unión económica. 
Los grupos intergubernamentalistas opinan que la integración regional no puede tener éxito si los Estados no la promueven. (Pimoljinda, 2010). Los factores importantes para intergubernamentalistas son el interés nacional y la soberanía (Pimoljinda, 2010). Hay países miembros de la CARICOM que quieren integración regional sin el abandono de su soberanía, porque consideran que su interés nacional vale más.

Los realistas piensan que la estabilidad y seguridad nacional son el factor clave para el establecimiento de organizaciones regionales (Pimoljinda, 2010). Los países miembros de la CARICOM son muy vulnerables. Ivelaw Griffith (2003), en su artículo "The Caribbean Security at the dawn of the 21st century: Continuity, Change, Challenge", destaca las vulnerabilidades y los retos de seguridad del Caribe como drogas, terrorismo, SIDA y conflictos fronterizos (territoriales y marítimos). Estas vulnerabilidades y retos de seguridad son otra razón para el establecimiento de la CARICOM y la necesidad de fortalecimiento y la profundización en la etapa de CSME.

Los liberales creen que la interdependencia económica aumentada es el factor prevalente en la integración regional (Pimoljinda, 2010). Es decir, que los liberales reconocen que ningún país tiene todos los recursos humanos, económicos y naturales. La interdependencia es una forma de supervivencia cotidiana para los países y aquellos que no quieren compartir/intercambiar sus recursos pueden sufrir pérdidas. Los Estados miembros de CARICOM creen que la interdependencia es la manera de proceder.

La CARICOM y CSME comparten metas comunes de integración. J. F. Hornbeck (2008), en su informe "CARICOM: Challenges and Opportunities for Caribbean Economic Integration", destaca estas metas comunes como la integración económica, la coordinación de políticas exteriores y la cooperación funcional (salud, educación, medio ambiente, ciencia y tecnología, entre otros). El objetivo básico para los miembros de la CARICOM es funcionar como una voz económica, política y regional en este mundo globalizado.

Los líderes de los Estados miembros de CARICOM reconocen que el logro de la unión política y económica es largo y difícil. Por eso, hay dos etapas para alcanzar esta meta. CEPAL (2009-2010), en su artículo, "Comercio e integración en el Caribe: tendencias y perspectivas", menciona las dos etapas de CSME. El período 2006-2009 es para el establecimiento del mercado único (CEPAL, 2009-2010). Por otro lado, el período 2010-2015 es para el establecimiento de la economía única (CEPAL, 2009-2010). Hay artículos del Revised Treaty of Chaguaramas de CARICOM para facilitar estas fases. El 33 enfatiza la eliminación de restricciones del derecho del establecimiento. El 37 destaca la eliminación 
de restricciones para la provisión de servicios. El 38 revela la eliminación de restricciones del sector financiero. El 40 destaca la eliminación de restricciones del movimiento del capital y transacciones corrientes. El 45 revela la libre movilidad de nacionales entre los Estados miembros. El 46 enfatiza la libre movilidad de nacionales capacitados entre los Estados miembros. El 86 destaca la libertad de tránsito.

A pesar de los esfuerzos de CARICOM para tener éxito, hay asimetrías de diferentes maneras que existen dentro de este esquema de integración regional. La Secretaría Permanente del SELA (2009), en su artículo, "Las asimetrías en los procesos de integración de América Latina y el Caribe", menciona cuatro tipos que existen en esquemas de integración: estructurales, comerciales, de políticas públicas e institucionales. Las asimetrías estructurales son difíciles de cambiar, imposibles y duran largo tiempo. Los ejemplos de estas, según el texto citado, son:

1. Las asimetrías comerciales.

i. El tamaño del mercado interno. El país con el más alto nivel de ingresos es Trinidad y Tobago. Haití es el país con la más baja tasa de ingresos. Haití es un país que tiene una historia de conflictos, altas deudas, corrupción y desastres naturales.

ii. Los recursos naturales. Ningún país tiene todos los recursos naturales en el mundo; pero hay algunos que tienen mucho más que otros. Trinidad y Tobago tiene petróleo y gas que contribuyen a casi la mitad de su PIB. Por otro lado, Granada tiene nuez moscada que contribuye en una manera significativa a su PIB. Los dos productos sufren los choques externos en el mundo; pero el petrolero y gas cuestan más que la nuez moscada $\mathrm{y}$, por eso, generan más dinero.

iii. El desarrollo de los sistemas implantados (en industrias de transformación, en finanzas, en ciencia y tecnología). Surinam es el país con la más alta tasa de innovación y tecnología. Es decir, que el gobierno y el sector privado han invertido mucho dinero en este país para elevar el nivel de innovación y tecnología. Haití es el país con la más baja tasa. Muchos grupos de negocios opinan que este país es demasiado inestable para invertir.

iv. El tamaño territorial. El Caribe es un área única porque hay países que son islas y otros que están en continente. Guyana es el Estado Miembro de CARICOM con la más grande área territorial. A pesar de esto, hay 
muchas áreas de Guyana que son inaccesibles y deshabitadas. Montserrat es el más pequeño país de CARICOM y sufría erupciones volcánicas terribles durante la década de los años noventa (Marín, 2013).

v. El nivel de capacitación de la mano de obra. La capacitación es un factor importante para cualquier país para sobrevivir la globalización y competir con otros mercados en el mundo. Sin la capacitación, la dependencia y la pobreza continuarán. Granada es el país con la más alta tasa de años de educación promedio. Haití es el país con la más baja tasa de años de educación promedio: 8.7 años. Armando López (2000, p. 2), en su artículo "La Comunidad del Caribe (CARICOM) en la encrucijada", menciona que la fuerza sin calificaciones es una característica de las economías subdesarrolladas como los países de CARICOM.

2. Asimetrías comerciales. Este tipo de asimetría es la calidad de comercio y la dependencia comercial. Países con bajos niveles de ingreso no tienen el dinero para invertir en innovación y tecnología para mejorar la calidad de comercio y deben depender de la inversión extranjera. Wendy Grenade (2011), en su artículo "Regionalism and Subregionalism in the Caribbean: Challenges and Prospects- Any Insights from Europe?", destaca que el proceso de CARICOM sufre fantasmas históricos. Es decir, que hay un legado de dependencia continua colonial. Graham Thomas y Esther Thomas (2009), en su artículo "The Impact of historical excess on the survival of the Caribbean Community and Common Market", mencionan que los países caribeños dependen de la ayuda extranjera. El fin de tratamiento preferencial y su impacto están discutidos adelante en este trabajo.

3. Asimetrías de políticas públicas. Hay diferentes políticas públicas a causa de las asimetrías, tales como la falta de voluntad política y las malas relaciones de poder entre los países miembros. Grenade (2011) menciona los retos de CARICOM, resultado de este tipo de asimetría: la falta de voluntad política, la ausencia de instituciones comunes y las relaciones de poder entre los países miembros de menor desarrollo y países de mayor desarrollo.

Existe falta de voluntad para implementar la Corte de Justicia Caribeña como la corte final de apelación. Según Grenade (2011), solo Guyana, Belice y Barbados tienen esta corte como su corte final de apelación. Los otros miembros no quieren esta institución como su corte final de apelación. Sin embargo, los Estados miembros de la Organización de Estados del Caribe Oriental tienen una corte similar: la Corte Suprema del Caribe Oriental que sirve como un ejemplo de la voluntad de los líderes de ese esquema de integración regional. 
La ausencia de instituciones comunes es otro reto de CARICOM. Hay países como Trinidad y Tobago y Jamaica que no quieren intercambiar su soberanía para la supranacionalidad. Estos países son muy nacionalistas y tienen miedo de la pérdida de soberanía que puede reducir su poder. Hay un vínculo entre este asunto y las malas relaciones de poder dentro del CARICOM. Muchas veces los países de mayor desarrollo opinan que los de menor desarrollo son cargas dependientes de sus recursos financieros y necesitan reducir su dependencia. Hace falta que estos países y sus líderes reconozcan que las asimetrías estructurales, como el tamaño territorial, los recursos naturales y el nivel de ingresos, son difíciles cambiar.

A pesar de esta perspectiva y la ausencia de instituciones comunes de CARICOM, existen instituciones de cooperación funcional de CARICOM. Estas instituciones son:

1. Agencia caribeña de respuesta a emergencia de desastres.

2. Instituto meteorológico caribeño.

3. Organización meteorológica caribeña.

4. Corporación caribeña de alimentación.

5. Instituto caribeño de la salud ambiental.

6. Instituto caribeño de desarrollo e investigación agrícola.

7. Centro regional caribeño para la educación y búsqueda de asistentes de salud veterinaria pública y de salud animal.

8. Asociación de parlamentarios de la Comunidad Caribeña.

9. Centro caribeño para la administración del desarrollo.

10. Instituto caribeño de nutrición y alimentación. ${ }^{2}$

2 Alberto Rocha Valencia, "La dimensión política de los procesos de integración regional y subregional de América Latina y el Caribe", en Jaime Preciado Coronado(Coord.), La integración politica latinoamericana y caribeña: Un proyecto comunitario para el siglo XXI, Asociación por la Unidad de Nuestra América, México, 2001, p. 185. 
Las instituciones asociadas son:

1. Banco caribeño de desarrollo.

2. Universidad de Indias Occidentales.

3. Instituto caribeño de leyes/ Centro de ICL.

4. Universidad de Guyana.

5. Organización de Estados del Caribe Oriental. ${ }^{3}$

Otras instituciones dentro de la CARICOM son:

1. Sistema de seguridad aérea del Caribe.

2. Centro del cambio climático de la Comunidad Caribeña.

3. Agencia de implementación para crimen y seguridad de CARICOM.

4. Corporación de alimentación caribeña.

5. Consejo de exámenes caribeños.

6. Organización caribeña de administradores tributarios.

7. Mecanismo regional para la industria pesquera del Caribe.

8. Instituto de información y traducción regional del Caribe.

9. Unión de telecomunicaciones del Caribe.

10. Comisión de competencia de CARICOM.

11. Consejo de educación legal. ${ }^{4}$ 
Es decir, hay más de veinte instituciones de cooperación funcional donde los Estados miembros de CARICOM participan y comparten.

4. Asimetrías institucionales. Esta categoría de asimetrías consiste en instituciones de orden interno (nacional) y orden regional. Está interrelacionada con la previa. Existen países que tienen diferentes prioridades. Algunos opinan que la diversificación es una. Otros piensan que el establecimiento de más instituciones supranacionales o más acuerdos con socios externos son prioridades relevantes. Hornbeck (2008) cree que el gran reto de CARICOM es cómo integrar un área diversa lograr los objetivos de países individuales y de CARICOM en una manera mutua y comprensiva sin negación de identidades nacionales y aspiraciones. Girvan (2009) opina que en CARICOM hay un marco institucional y gubernamental que es débil. La debilidad de este marco puede prevenir la transformación de este esquema de integración regional del mercado común a la unión política y económica.

López (2000) opina que CARICOM tiene éxitos y fallas de cooperación funcional. Las áreas en que ha fallado son: cultura, transporte y representación caribeña. Girvan (2009) comparte esta perspectiva y cree que hay falta de una identidad cultural común. Los dos teóricos no reconocen las asimetrías ideológicas porque cada país miembro de CARICOM es diferente. Guyana y Trinidad y Tobago son emblemáticos de este asunto, porque los dos países son multiétnicos. La supranacionalidad ha contribuido a problemas con transporte y la representación caribeña.

El éxito que López (2000) ve en CARICOM está en las áreas de cooperación funcional como la educación y la salud. La Universidad de Las Indias Occidentales es un gran éxito porque hay universidades en Barbados, Jamaica y Trinidad y Tobago y sucursales en otros países miembros. La Universidad de Guyana es otro ejemplo del éxito de cooperación funcional en educación. En el área de salud, hay el Instituto caribeño de nutrición y alimentación.

El fin del tratamiento especial es una oportunidad y, a la vez, es un reto de CARICOM. Es una oportunidad porque señala el fin del síndrome colonial de dependencia que muchos países de este esquema sufren. Adicionalmente, es un reto porque, según las asimetrías estructurales, los países de CARICOM no pueden sobrevivir y enfrentar este nuevo sistema de independencia o interdependencia.

Clive Thomas (2008), en su artículo, "CARICOM Perspectives on the CARIFORUM-EC, Economic Partnership Agreement”, menciona el propósito y retos de acuerdo de asociación comercial. Este autor destaca que el acuerdo de 
asociación comercial es un documento y un instrumento para promover los objetivos específicos de desarrollo. Hay seis condiciones que son 'garantías' para CARICOM con este nuevo acuerdo.

1. Creación de comercio después de la eliminación de barreras al comercio.

2. Reformas desarrollistas.

3. Integración y desarrollo regional sostenible.

4. Oportunidad de erradicar pobreza y otros males sociales.

5. Comercio al servicio del desarrollo.

6. Reformas económicas.

Thomas (2008) destaca los planes para prevenir y 'proteger' los países del África, Caribe y Pacífico que previamente tienen acuerdos comerciales de tratamiento preferencial para desastre económico. Estos planes son el mejoramiento de reglas de origen para CARIFORUM, las fases para las negociaciones, los mecanismos de proteger industrias como nombramiento de industrias / sectores sensitivos y el tratamiento 'cero por cero' de subsidios agrícolas y no vincular acceso de oportunidades del mercado para la Unión Europea (Thomas, 2008).

Las fuentes de los problemas que Thomas (2008) ve con este acuerdo son numerosas. En primer lugar, hay evidencia de que no apoya la teoría del acuerdo de asociación comercial. La teoría y la práctica no están interrelacionadas porque existen disparidades. El apunte de este nuevo acuerdo puede provocar problemas serios. Anthony Peter Gonzales (2002) en su artículo, "CARICOM, the European Union and International Linkages in External Trade", menciona las circunstancias posibles: conflictos laborales, pugnas étnicas, más altos costes de transporte de pequeños volúmenes, más dificultades con diversificación a causa de los pequeños mercados y altos costes de transacción para las empresas pequeñas que quieren comerciar con mercados exteriores.

Otra fuente del problema es que hay disputas sobre las evaluaciones e interpretaciones. Hay personas que tienen diferentes interpretaciones. Algunos creen que es un acuerdo que genera competitividad y diversificación, que son asuntos esenciales para sobrevivir la globalización. A la vez, algunos creen que es un intento de socavar los mercados vulnerables de los países subdesarrollados y 
pueden provocar caos, como los problemas mencionados por Gonzales (2002). Estas disputas señalan la necesidad de más evaluaciones.

Otro problema son los bajos niveles de conocimiento y participación. Thomas (2008) menciona que hay muchas reuniones sobre este acuerdo y los participantes carecen de conocimiento de este acuerdo. La carencia de participación representa una falta de interés de asuntos que aquejan la región ACP y que los países hegemónicos como los de la Unión Europea pueden continuar su hegemonía sobre estas regiones subdesarrolladas del mundo. La falta de participación socava la voz de estos países vulnerables. La falta de conocimiento señala la carencia de poder para escoger y hacer decisiones esenciales.

Vincent J. Atkins (2000) en su artículo, "Regional agricultural policy processes in the Caribbean economy", cree que la reciprocidad no puede ayudar los países vulnerables como los de ACP. La reciprocidad solo beneficia la Unión Europea porque muchas veces las asimetrías estructurales de los países miembros de CARICOM no les permiten a estos países elevar su nivel de participación en el mercado. Atkins no es el único autor que opina que la reciprocidad solo beneficia la Unión Europea y sofoca los mercados de CARICOM y los otros países de ACP. Girvan (2009) plantea que Europa global hizo este acuerdo comercial para proteger sus inversiones europeas, fortalecer los derechos de propiedad intelectual y reducir las barreras no arancelarias. Con la llegada de la Organización Mundial del Comercio, se llegaría al fin del tratamiento preferencial de los países de ACP.

CARICOM reconocía sus retos y establecía la Comisión de Competencia Caribeña. Esta Comisión promueve competición dentro de los países miembros $\mathrm{y}$, a la vez, protege las empresas o sectores vulnerables de competición desfavorables. Es decir, esta Comisión puede ayudar a los países y a los sectores vulnerables a reducir su dependencia de la inversión extranjera directa, cambiar su perspectiva de dependencia a interdependencia y competitividad y, a la vez, proteger estos países de prácticas desfavorables. Taimoon Stewart (2000), en su artículo "Developing and Implementing a Competition Regime: Challenges Faced by Small Open Economies", destaca los retos que pueden prevenir la eficiencia de esta Comisión de CARICOM. Estos retos son el desarrollo de recursos humanos, el desarrollo de la cultura de competición, los recursos financieros y la formación de personal de derecho para promulgar leyes. 


\section{Las oportunidades para CARICOM}

Hay otras oportunidades que existen para ayudar a que este esquema de integración regional sea poderoso en el mundo. Hornbeck, Atkins, Graham y Esther Thomas y CEPAL opinan que la diversificación puede solventar los problemas económicos de CARICOM. CEPAL (2009-2010) destaca que la diversificación de las economías de CARICOM creará nuevas ventajas comparativas.

Atkins (2000) opina que los subsidios ayudarían a los productos no tradicionales como ganado, productos lácteos, aves domésticas y arroz. (114) Sin embargo, este autor reconoce que hay pocos recursos para la diversificación de las economías de los Estados miembros de CARICOM. Adicionalmente, Atkins (2000) destaca que el primer intento de diversificar las economías de este esquema de integración regional recibió un enfrentamiento de la Organización de Cooperación y Desarrollo Económico que opinó que había sido una competición de impuestos dañosos. CEPAL (2009-2010) revela los países que están diversificando sus economías: Barbados, Granada, Guyana, Jamaica, Santa Lucía, San Vicente, las Granadinas, y Trinidad y Tobago. La siguiente tabla 3 muestra los productos y servicios del proceso de diversificación.

\section{Tabla 3}

Áreas de diversificación de economías de CARICOM

\begin{tabular}{|c|c|}
\hline País & Áreas de diversificación \\
\hline Barbados & algodón \\
\hline Granada & $\begin{array}{l}\text { agua mineral y gaseosa, mermelada, jalea, sirope, licores y un cal- } \\
\text { mante basado en nuez moscada }\end{array}$ \\
\hline Guyana & $\begin{array}{l}\text { frutas, vegetales, producción ganadera, acuicultura, observación } \\
\text { de aves, ecoturismo, deporte de vela y telecomunicaciones. Áreas } \\
\text { propuestas de diversificación: alternativas de energía- hidroeléctri- } \\
\text { ca y biocombustibles. }\end{array}$ \\
\hline Jamaica & zona de libre comercio en la bahía de Montego. \\
\hline Santa Lucía & orquídeas, tejidos, industria pesquera, carne, acuicultura. \\
\hline $\begin{array}{l}\text { San Vicente y las } \\
\text { Granadinas }\end{array}$ & mango, palta, plátano, camote, taro, chile. \\
\hline Trinidad y Tobago & $\begin{array}{l}\text { metanol, amoniaco, urea, instalaciones para conferencias y habita- } \\
\text { ciones de hotel, transporte aéreo y marítimo. Tobago- promoción } \\
\text { de mercado como destino de buceo y ecoturismo. }\end{array}$ \\
\hline
\end{tabular}

Nota: Elaboración propia con base de datos de CEPAL, 2009-2010).

Graham Thomas y Esther Thomas (2009) opinan que CARICOM debe desarrollar un mercado agresivo que permita un producto único turístico (ecoturismo, 
turismo sanitario). Sin embargo, CEPAL (2009-2010) hace un buen trabajo con las siguientes limitaciones de diversificación:

1. Oferta.

2. Falta del acceso al mercado.

3. Escasa respuesta a preferencias no recíprocas se ha provocado las restricciones de la oferta.

4. Altos costes de energía y telecomunicaciones.

5. El acceso inadecuado al capital.

6. Bajos niveles de innovación.

7. El desarrollo insuficiente del sector empresarial.

8. La debilidad de instituciones.

9. El escaso desarrollo de los mercados financieros.

Hornbeck (2008) puede añadir una limitación a la diversificación: las economías de CARICOM no pueden desarrollarse bastante rápido para mitigar problemas.

\section{Las realidades de los países miembros de CARICOM}

Ya se ha discutido que los países miembros de CARICOM sufren asimetrías. Esta realidad muestra la necesidad del fortalecimiento de la integración regional. Chad Bown et al. del Banco Mundial (2017), en su libro Mejores vecinos: Hacia una renovación de la integración económica en América Latina, revela las ventajas de la integración regional. Esta investigadora va a vincularlas con las realidades y las asimetrías de los países miembros de CARICOM.

En primer lugar, la integración regional impulsa el crecimiento (Bown et al., 2017). La tasa del crecimiento del PIB no es la misma en cada país miembro. Por eso, la integración regional es imprescindible. Según la tabla adjunta, Haití tiene el más pequeño mercado de CARICOM. Este tamaño pequeño agrava más 
la situación y el legado de pobreza, dictadura y deuda externa del país. Por otro lado, en Trinidad y Tobago, el mercado es más grande y no sufre los mismos desafíos de Haití. Un esquema de integración regional fortalece a los miembros trabajadores; pero débiles.

El mejoramiento de la conectividad es otra ventaja imprescindible en esta investigación. Bown (2017) revela que la integración regional mejora la conectividad (la calidad de infraestructura y la conectividad). La asimetría del desarrollo de sistemas implantados afecta más a Haití que a los otros países miembros. Los fondos generados en los esquemas de integración regional están invertidos en el mejoramiento de la calidad de vida de los grupos más vulnerables. En países de bajo desarrollo como Haití, la conectividad es una necesidad especialmente en este mundo globalizado. Los países sin medianos ni altos niveles de desarrollo experimentan retrasos en su progreso.

Nuestras diferencias nos unen. En este mundo diverso y globalizado, las diferencias pueden unirnos en vez de provocar pugnas. Bown (2017) destacan que el gusto está en la diferencia (complementar acuerdos comerciales). Los países miembros de CARICOM tienen diferentes recursos naturales. La necesidad de intercambiar es más vital entre los países que experimentan estas diferencias. Por ejemplo, Trinidad y Tobago es el país miembro con altas cantidades de petróleo y gas. Existen acuerdos entre otros países miembros y Trinidad y Tobago con respecto a la compra y la venta de estos productos. Sin embargo, los países miembros de OECO compran este producto de Venezuela, con la iniciativa de ALBA. Aunque no hay pugnas entre los países miembros a causa de esta decisión de OECO, esta es una tensión posible en los años que vienen.

Ser económicamente más competitiva con el resto del mundo es otra ventaja (Bown, 2017). La asimetría de política pública contribuye a la falta de competitividad dentro de los esquemas de integración regional. La falta de voluntad política al rechazar pugnas y la decisión de trabajar juntos a pesar de los retos que surgen tardan los procesos de desarrollo económico y desarrollo humano en CARICOM. Los países de otros esquemas de integración regional experimentan retos, pero están más dedicados y listos a enfrentarlos. No es un caso de la supervivencia de los más aptos: Trabajan juntos para construir un futuro más seguro para las generaciones venideras.

\section{Recomendaciones}

Muchos estudios han ofrecido recomendaciones para solventar los retos que las economías pequeñas y vulnerables de CARICOM enfrentan. Marín (2013) 
recomienda que los esquemas de integración consideren, las brechas de desarrollo y competitividad y reclama la necesidad de impulsar una armonización en las políticas de incentivos dirigidos a la atracción de inversiones y a la promoción de exportaciones.

Otra recomendación es la integración eficaz para mitigar el tamaño de la región. La integración es un proceso difícil. De hecho, el Secretario General de CARICOM, Irwin La Rocque (2013), en su discurso "Regional Integration Process and Vision for the future of CARICOM", menciona que la integración es gradual y lenta. No es fácil unir los países de diferentes culturas, grupos étnicos, niveles de ingresos, índices de desarrollo humano y demografía. A pesar de esto, la integración es panacea a muchos problemas que países vulnerables como los de CARICOM enfrentan.

La implementación de las políticas de desarrollo sostenible coherentes es otra recomendación. La coherencia de las políticas es un problema en cualquier esquema de integración regional. Hay líderes que tienen diferentes perspectivas y malinterpretaciones de asuntos. Ya se ha mencionado que en la Federación de las Antillas, los dos primeros ministros tenían diferentes perspectivas del mismo asunto.

La Rocque (2013) también ofrece recomendaciones a CARICOM para profundizar la integración regional. En primer lugar, él cree que CARICOM necesita aprender las lecciones de OECO. Este esquema es el mejor ejemplo para CARICOM emular. La Rocque no es la única persona que opina esto. CEPAL (2009-2010) tiene la misma perspectiva y enfatiza las instituciones comunes y supranacionales de OECO que son: un banco central, bolsa de valores, magistratura, sistema de seguridad, servicio conjunto de compras de productos farmacéuticos, misiones diplomáticas conjuntas y un organismo regulador de aviación civil.

La expansión de comercio y la promoción de competitividad es otra recomendación que el Secretario General ofrece. Hay muchas oportunidades para explorar por los países de CARICOM. Desafortunadamente, no todos los países de este esquema pueden explorar ni quieren estas oportunidades en este mundo globalizado.

El establecimiento de metas es otra recomendación del Secretario General (La Rocque, 2013). Él opina que hay que establecer objetivos que tomen en cuenta la necesidad, la urgencia y la capacidad. Es decir, que estas metas sean realistas, relevantes y puedan ayudar a CARICOM a lograr su último objetivo. Sin metas, no hay camino para el destino y no hay esperanza. 
La toma de decisiones eficaz es otra recomendación de La Rocque. Las decisiones como las metas necesitan ser relevantes, realistas y tienen la habilidad de eliminar los obstáculos que previenen este esquema para tener éxito como OECO. La falta de decisiones eficaces puede dañar más economías pequeñas, frágiles y vulnerables.

La Corte de Justicia Caribeña es una institución supranacional de CARICOM cuyo objetivo es conciliar disputas entre países y ciudadanías. Desafortunadamente, hay países que no quieren tener esta corte como su corte final de apelación. La Rocque (2013) recomienda que los Estados miembros tomen la decisión de tener esta corte como su corte final de apelación. Esta corte es eficaz y solventó una disputa entre Shanique Myrie, jamaicana y la migración de Barbados.

Otra recomendación de CEPAL (2009-2010) es la implementación de la economía única. La economía única fortalecerá las economías frágiles, vulnerables y pequeñas de este esquema de integración regional. Desafortunadamente, la implementación de este tipo de economía sufre muchas barreras. La barrera más importante es la falta de voluntad política para implementar esta decisión.

En tercer lugar, CEPAL (2009-2010) recomienda que CARICOM necesita alentar a la Unión Europea a acelerar el desembolso de fondos para promover integración. Esta es una buena recomendación; pero, al igual que los países líderes miembros de CARICOM, es posible que los de los Estados miembros de la Unión Europea también falten a la voluntad política de ejecutar decisiones. La Unión Europea sufre choques externos y asimetrías estructurales. Adicionalmente, CARICOM no tiene el poder económico, político e ideológico para estimular estas decisiones de la Unión Europea.

\section{Conclusiones}

No hay duda de que la CARICOM ha evolucionado. En cada nivel de integración regional, la CARICOM, como los otros esquemas de integración regional, ha experimentado retos y oportunidades. Los retos no son obstáculos porque provocan el fortalecimiento de los Estados miembros para enfrentar sus debilidades y amenazas. Este mundo es una aldea global en donde el establecer y el fortalecer vínculos entre países son acciones esenciales. La interdependencia forma parte de la agenda política de muchas administraciones. En la misma manera, la integración regional fortalece los países más vulnerables. Por eso, las asimetrías entre los países promueven la necesidad de integración regional. La unidad es la única manera de sobrevivir la globalización, porque es un caso 
de la supervivencia de los más aptos. El OECO y la Unión Europea son emblemáticos de la integración regional.

Los países pequeños son más vulnerables a la globalización que los países grandes. El fin del tratamiento especial es un golpe adicional a estos países y, a la vez, una oportunidad de terminar su estado de dependencia e iniciar la interdependencia. Los Estados miembros de CARICOM son pequeños y vulnerables. Estos países subdesarrollados sufren muchas asimetrías, pero hay oportunidades que están disponibles para elevar sus niveles de desarrollo económico, social y humano. Los Estados miembros de CARICOM necesitan explorar estas oportunidades y prestar atención a las recomendaciones de los estudios teóricos caribeños para lograr sus objetivos. Estos parten de la realidad y son los más aptos para ofrecer soluciones relevantes.

Una recomendación importantísima es la necesidad de establecer órganos supranacionales. Los órganos supranacionales fortalecen y avanzan el proceso de integración regional. El proceso de la unidad en este esquema de integración regional es largo y existe la necesidad de elevar el tono del debate. Es la hora de promover cooperación y rechazar pugnas que solo sirven para debilitar más las economías y disminuir el desarrollo humano en los Estados miembros de CARICOM. El proverbio chino dice: "Un viaje de mil millas comienza con un solo paso". Este paso es la unidad en el camino. 


\section{Referencias}

Atkins, Vincent J. (2000). Regional agricultural policy processes in the Caribbean Community. En Agricultural policy networking: the way forward (pp. 113124)Wageningen: Technical Centre for Agricultural and Rural Cooperation (CTA). London: Sayce Publishing.

Barboza, Jorge. (2010). Procesos de unión económica y monetaria en Centroamérica. Ponencia Lanzamiento de la Revista de Derecho Comunitario, Internacional y Derechos Humanos. Universidad de Costa Rica, Costa Rica. Recuperado de http://www.derechocomunitario.ucr.ac.cr/index. php?option $=$ com_content $\&$ view $=$ article\&id=116\%3Aunion-economica-monetaria-cabarboza $\&$ catid $=28 \% 3 \mathrm{~A} 1 \&$ Itemid $=27$

Bown, Chad (2017). Mejores vecinos: Hacia una renovación de la integración económica en América Latina (Resumen). Washington, DC: Banco Mundial.

CARICOM. (2010). Tratado Revisado de Chaguaramas. Recuperado dehttp://www. caricom.org/jsp/community/revised_treaty-text.pdf

CEPAL. (2009-2010). Comercio e integración en el Caribe: Tendencias y perspectivas. En Panorama de la inserción internacional de América Latina y el Caribe 2009-2010: Crisis originada en el Centro y recuperación impulsada por las economías emergentes (pp.123-150) Recuperado de http://www.eclac.cl/ publicaciones/xml/5/40695/Comercio_integracion_Caribe_tendencias_Capitulo_IV.pdf

Fontes, Max. (2000). Mercosul and Supranationality: How to Overcome Brazilian Constitutional Obstacles. Recuperado de http://www.hottopos.com/harvard $4 /$ max.htm

Girvan, Norman. (2005). Reinterpreting the Caribbean. In The Caribbean Economy: A Reader. Kingston: Ian Randle. (pp.1-17)

Girvan, Norman. (2009). The Caribbean Community encounters Global Europe Implications of Economic Partnership Agreements between CARIFORUM and EU. En Alfredo Guerra-Borges (Coord.), Fin de época: De la integración tradicional al regionalismo estratégico (pp. 352-381) México: Siglo Veintiuno Editores.

Gonzales, Anthony. (2002). CARICOM, the European Union and International Linkages in External Trade. Jean Monnet/Robert Schuman Paper Series, 2(4), 16. Recuperado de http://aei.pitt.edu/8082/1/gonzalesworkingpaper.pdf

Grenade, Wendy. (2011). Regionalism and sub regionalism in the Caribbean: Challenges and Prospects-Any insights from Europe? Jean Monnet/Robert Schuman Paper Series, 11(4), 25. Recuperado de http://aei.pitt.edu/33484/1/ GrenadeCaribRegionalismLong2011edi.pdf 
Griffith, Ivelaw. (2003). The Caribbean security scenario at the dawn of the 21st century: continuity, change, challenge. North- South Agenda Papers, 65.

Hornbeck, J. F. (2008). CARICOM: Challenges and Opportunities for Caribbean Economic integration. Congressional Regional Service Report for U.S. Congress: Recuperado de http:/www.sice.oas.org/TPD/CAR_EU/Studies/CRSCARICOM_Challenges_e.pdf

La Rocque, Irwin. (2013). Regional Integration Process and Vision for the future of CARICOM. Recuperado de http://caricom.org/media-http://caricom.org/ media-center/communications/speeches/distinguished-lecture-by-ambassador-irwin-larocque-secretary-general-caribb

López, Armando. (2000). La Comunidad del Caribe (CARICOM) en la encrucijada. Recuperado de https://www.nodo50.org/cubasigloXXI/economia/ coll1_310503.pdf

Marín, Dunnia. (2013). El abordaje de las asimetrías estructurales en los esquemas de integración regional. Heredia, Costa Rica: Universidad de Gestión Editorial de la Escuela de Relaciones Internacionales.

Pimoljinda, Thanawat. (2010). Theoretical Discussion on Regional Integration: EU-ASEAN Perspective. Recuperado de http://www.academia.edu/388618/ Theoretical_Discussion_on_Regional_Integration_EU-ASEAN_Perspective

Rocha Valencia, Alberto. (2001). La dimensión política de los procesos de integración regional y subregional de América Latina y el Caribe. En Jaime Preciado Coronado (Coord.), La integración política latinoamericana y caribeña: Un proyecto comunitario para el siglo XXI (pp. 165-212). México: Asociación por la Unidad de Nuestra América.

Secretaría Permanente del SELA. (2011). Las asimetrías en los procesos de integración en América Latina y el Caribe. Recuperado de http://www.sela.org/media/266783/t023600003433-0-tratamiento_de_las_asimetrias_en_los_procesos_de_integracion_en_alc.pdf

Stewart, Taimoon. (2000). Developing and Implementing a Competition Regime: Challenges Faced by Small Open Economies. Boletín latinoamericano de competencia, 11, 5. Recuperado de http://ec.europa.eu/competition/publications/blc/boletin_11_1_es.pdf

Thomas, Clive. (2008). CARICOM Perspectives on the CARIFORUM-EC, Economic Partnership Agreement. Recuperado de http://www.normangirvan. info/wp-content/uploads/2008/05/clive-thomas-caricom-perspective-onthe-cf-ec-epa-may-2008.pdf

Thomas, Graham y Esther Thomas. (2009). The Impact of Historical Excess on the Survival of the Caribbean Community and Common Market. Tamara Journal, 8(8.2), 11. Recuperado de https://tamarajournal.com/index.php/tamara/ article/view/318/298 